\title{
Knockdown of AQP1 inhibits growth and invasion of human ovarian cancer cells
}

\author{
YANLI WANG $^{1}$, YANYAN FAN ${ }^{1}$, CHUNHUA ZHENG $^{1}$ and XIAOMENG ZHANG ${ }^{2}$ \\ ${ }^{1}$ Department of Gynecology, The First Hospital of Jilin University, Changchun, Jilin 130021; \\ ${ }^{2}$ Department of Ophthalmology, The Second Hospital of Jilin University, Changchun, Jilin 130041, P.R. China
}

Received June 26, 2016; Accepted April 24, 2017

DOI: $10.3892 / \mathrm{mmr} .2017 .7282$

\begin{abstract}
Aquaporin 1 (AQP1), which is a water channel protein, has been demonstrated to have an important role in cell proliferation and migration of various cancers. However, its specific role in ovarian cancer remains to be elucidated. The present study demonstrated that AQP1 expression was elevated in the majority of patients with ovarian cancer compared with normal ovarian tissues. In addition, a short interfering (si)RNA targeting AQP1 was established, and transfected into the SKOV3 ovarian cancer cell line, to investigate the effects on cell viability, apoptosis, migration and invasion in the ovarian cancer cells using an MTT assay, flow cytometry, wound healing and Transwell invasion chamber assays, respectively. The results of the present study demonstrated that siRNA targeting AQP1 effectively downregulated AQP1 expression at the mRNA and protein levels, markedly suppressed cell viability, migration and invasion and promoted apoptosis of ovarian cancers cells. These results suggested that AQP1 may serve as a novel target for ovarian cancer treatment in the future.

\section{Introduction}

Ovarian cancer is the gynecological malignancy associated with the greatest risk of mortality (1), due to its undetectable characteristics until a late stage and poor prognosis (2). Ascite formation is frequently detected during progression of the disease and ascite volume is associated with poor prognosis of ovarian cancer (3). The underlying mechanisms of peritoneal ascite development have not been well-elucidated. It has previously been suggested that osmotically active proteins expressed in tumor mass or adjacent tissues, induced enhanced
\end{abstract}

Correspondence to: Dr Xiaomeng Zhang, Department of Ophthalmology, The Second Hospital of Jilin University, 218 Ziqiang Street, Changchun, Jilin 130041, P.R. China

E-mail: zhangxm15106@sina.com

Key words: ovarian cancer, aquaporin 1, proliferation, apoptosis, migration, invasion capillary penetration of water and thus contributed to extensive fluid accumulation within the peritoneal cavity (4-6).

The Aquaporins (AQP) are a family of highly conserved small ( $30 \mathrm{kd} /$ monomer) membrane channel proteins that selectively transport water and small molecules $(7,8)$. Currently, 13 homologous AQPs have been identified in mammals (9). Of these proteins, AQP1 is the one of the first that was identified, and has been revealed to be highly expressed in the microvessel endothelium (10), and strictly selective for water transport (11). Saadoun et al (12) reported that the water permeabilization results in increased hydrostatic pressure locally, leading to polarization of AQP1. AQP1 additionally increases permeability of the blood-brain barrier and increases water flow, contributing to brain tumor edema (13). Inhibition of AQP1 results in alveolar-capillary osmotic water permeability in lungs $(14,15)$. Recently, the roles of ion channels including AQP1 have been hypothesized to be associated with various cancer types with a contribution to cellular proliferation and tumor migration (16-20). AQP1 has previously been demonstrated to act as a novel biomarker for aggressive ovarian cancer, and an independent marker for prognosis (21). However, the biological role of AQP1 in ovarian cancer cell viability, apoptosis, migration and invasion remains to be fully elucidated.

The present study aimed to investigate the biological function of AQP1 on cell viability, apoptosis, migration and invasion in ovarian cancer cells. It was demonstrated that downregulation of AQP1 effectively suppressed cell viability, migration and invasion, and induced cell apoptosis in ovarian cancer cells. These findings will contribute to future research, with AQP1 as a novel target for gene therapy in the treatment of ovarian cancer in the future.

\section{Materials and methods}

Patients and tissue samples. The present study was approved by the Ethics Committee of Jilin University (Changchun, China). Clinical samples of 46 cases of ovarian serous tumor tissue and 10 cases of normal ovary tissue were collected from The First Affiliated Hospital of Jilin University from January 2013 to December 2014. All samples were immediately frozen in liquid nitrogen, and stored at $-80^{\circ} \mathrm{C}$ until RNA extraction. Informed consent was obtained from all patients. 
The mean age of the patients was 58 (range, 38-83 years). Of 46 cases of malignant tumors, 12 were highly differentiated, 18 moderately differentiated and 16 poorly differentiated, based on the classification of pathological differentiation degree. A total of 14 cases were stage I, 18 were stage II and 14 were stage III-stage IV based on the International Federation of Gynecology and Obstetrics (FIGO) stage classification. In addition, there were 18 cases with lymph node metastasis and 28 cases without lymph node metastasis. A total of 36 cases were diagnosed with ascites (>50 ml fluid) following abdominocentesis or abdominal surgery.

Immunochemistry staining. All samples were fixed with $4 \%$ paraformaldehyde for $48 \mathrm{~h}$ at room temperature, and embedded in paraffin at room temperature. Serial sections were collected at a thickness of $4 \mu \mathrm{m}$. The immunohistochemistry staining procedures were performed using standard protocols. Paraffin sections were deparaffinized in xylene for $15 \mathrm{~min}$ and rehydrated in graded ethanol $(100,95,85,70$ and 50\%) at room temperature. Heat induced antigen retrieval was performed using citrate buffer. Quenching was performed using 3\% hydrogen peroxide for $15 \mathrm{~min}$. Non-specific antibody binding was blocked by incubation with goat serum (Sigma-Aldrich; Merck KGaA, Darmstadt, Germany) for $30 \mathrm{~min}$ at room temperature. Sections were then incubated with mouse monoclonal anti-AQP1 antibody (1:1,000 dilution; cat. no. sc-32738; Santa Cruz Biotechnology, Inc., Dallas, TX, USA) diluted with PBS overnight at $4^{\circ} \mathrm{C}$. A biotin-conjugated polyclonal goat anti-mouse antibody (1:5,000 dilution; cat. no. sc-2039; Santa Cruz Biotechnology, Inc.) was added for $30 \mathrm{~min}$ at room temperature. The visualization of AQP1 expression was achieved using an avidin-biotin-peroxidase kit (Sigma-Aldrich; Merck KGaA) and followed by diaminobenzidin (DAKO; Agilent Technologies, Inc., Santa Clara, CA, USA) staining at room temperature for $30 \mathrm{~min}$. All results were observed under a light microscope (X51; Olympus Corporation, Tokyo, Japan). Samples with AQP1 expression in $>25 \%$ tissues were regarded as positive samples and $<25 \%$ were negative.

Cell culture and transfection. The SKOV3 human ovarian cancer cell line was purchased from the Type Culture Collection of the Chinese Academy of Sciences (Shanghai, China). SKOV3 cells were cultured in RPMI-1640 medium (Gibco; Thermo Fisher Scientific, Inc., Waltham, MA, USA) supplemented with $10 \%$ (v/v) fetal bovine serum (FBS; HyClone, GE Healthcare Life Sciences, Logan, UT, USA), $100 \mathrm{U} / \mathrm{ml}$ penicillin and $100 \mathrm{mg} / \mathrm{ml}$ streptomycin in a humidified atmosphere containing $5 \% \mathrm{CO}_{2}$ at $37^{\circ} \mathrm{C}$.

Small interfering (si)RNA against AQP1 (si-AQP1; 5'-GAGGCTGATTCCTCTCATTTC-3') and siRNA negative scramble control (si-NC; 5'-CCTAAGGTTAAGTCGCCC TCG-3') were obtained from Shanghai GenePharma Co., Ltd (Shanghai, China), and were transfected at a final concentration of $100 \mathrm{nM}$ into SKOV3 cells $\left(1 \times 10^{5}\right.$ cells/well) in RPMI-1640 medium using Lipofectamine 2000 (Invitrogen; Thermo Fisher Scientific Inc.) at room temperature according to the manufacturer's protocol. Transfection efficiencies were determined in every experiment at $48 \mathrm{~h}$ following transfection using reverse transcription-quantitative polymerase chain reaction (RT-qPCR) and western blotting.
$R T-q P C R$. Total RNA was isolated from cultured cells or tissues using TRIzol ${ }^{\circledR}$ (Invitrogen; Thermo Fisher Scientific, Inc.) according to the manufacturer's protocol. Reverse transcription was performed using RevertAid ${ }^{\mathrm{TM}}$ first strand cDNA synthesis kit (Fermentas; Thermo Fisher Scientific, Inc.). qPCR was performed using SYBR Premix Ex Taq (Takara Bio, Inc., Otsu, Japan) in an ABI 7900 Fast system (Applied Biosystems; Thermo Fisher Scientific, Inc.). The following primers were used: AQP1, forward 5'-GCCATTTAGAGG GTGAAG-3', reverse 5'-TGACAAGAGGGAGTAGAG-3'; and GAPDH, forward 5'-CACCCACTCCTCCACCTTTG-3' and reverse 5'-CCACCACCCTGTTGCTGTAG-3', as previously described (22). The PCR conditions were as follows: An initial denaturing step at $95^{\circ} \mathrm{C}$ for $3 \mathrm{~min}$, followed by 40 cycles of denaturation at $95^{\circ} \mathrm{C}$ for $20 \mathrm{sec}$, annealing/extension at $60^{\circ} \mathrm{C}$ for $30 \mathrm{sec}$ and final extension step at $72^{\circ} \mathrm{C}$ for $10 \mathrm{~min}$. GAPDH was used as an internal standard to normalize the AQP1 expression level using the $2^{-\Delta \Delta \mathrm{Cq}}$ method (23).

Western blot analysis. Cultured cells were collected and washed twice with PBS and lysed in ice-cold radioimmunoprecipitation assay buffer (JRDun Biotechnology, Co., Ltd., Shanghai, China) containing $0.01 \%$ protease inhibitor cocktail (Sigma-Aldrich; Merck KGaA) and incubated on ice for $30 \mathrm{~min}$. Protein concentration was determined using a bicinchoninic acid assay kit (Pierce; Thermo Fisher Scientific, Inc.). A total of $20 \mu \mathrm{g}$ protein was separated by $10 \%$ SDS-PAGE and then transferred to polyvinylidene difluoride membranes (EMD Millipore, Billerica, MA, USA). The membranes were then blocked with $5 \%$ non-fat milk for $2 \mathrm{~h}$ at room temperature to block nonspecific binding. The membranes were subsequently incubated overnight at $4^{\circ} \mathrm{C}$ with mouse monoclonal anti-AQP1 antibody (1:1,000 dilution; cat. no. sc-32738) and mouse monoclonal anti-GAPDH antibody (1:10,000 dilution; cat. no. sc-32233) (both from Santa Cruz Biotechnology, Inc.). The membranes then were incubated with goat-anti-mouse horseradish peroxidase-conjugated immunoglobulin $\mathrm{G}$ (1:10,000 dilution; cat. no. sc-2204; Santa Cruz Biotechnology, Inc.) at room temperature for $1 \mathrm{~h}$. GAPDH was used as an internal control. The protein bands were visualized using a super-signal chemiluminescence detection ECL kit (Pierce; Thermo Fisher Scientific, Inc.).

Cell viability assay. Cell viability was determined by 3-(4,5-dimethylthiazol-2-yl)-2,5-diphenyltetrazolium bromide (MTT) assay. Briefly, the cell density of transfected cells was adjusted to $5 \times 10^{4} / \mathrm{ml}$, and cells were added to a 96-well plate $(100 \mu \mathrm{l} /$ well) and cultured for $24-72 \mathrm{~h}$. At the indicated time (24, 48 and $72 \mathrm{~h}$ post-transfection), $20 \mu \mathrm{l}$ MTT solution ( $5 \mathrm{mg} / \mathrm{ml}$, Sigma-Aldrich; Merck KGaA) was added to each well followed by incubation at $37^{\circ} \mathrm{C}$ for $4 \mathrm{~h}$. Then, centrifugation was performed at $2,000 \mathrm{x}$ g for $10 \mathrm{~min}$ at room temperature. The supernatant was removed, and $200 \mu \mathrm{l}$ dimethyl sulfoxide (Sigma-Aldrich; Merck KGaA) was added for $10 \mathrm{~min}$ at $37^{\circ} \mathrm{C}$. The absorbance in each well was measured at a wavelength of $570 \mathrm{~nm}$ using an ELISA multi-well spectrophotometer (Molecular Devices, LLC, Sunnyvale, CA, USA).

Apoptosis analysis. SKOV3 cells $\left(2 \times 10^{5}\right.$ cells) were seeded in 6-well plates in RPMI-1640 medium containing 10\% FBS 
A

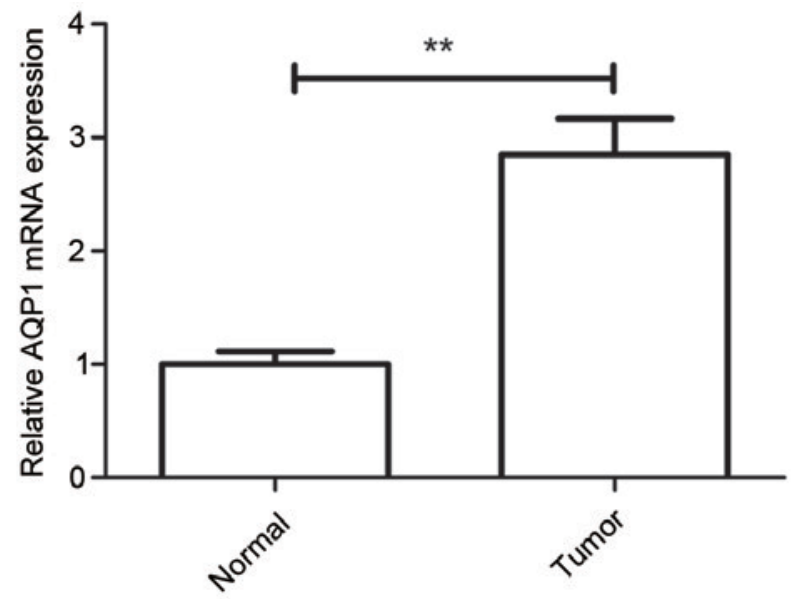

B

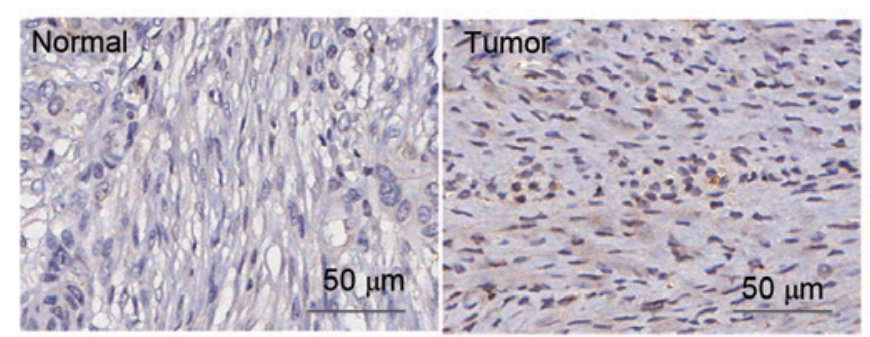

Figure 1. Levels of AQP1 are increased in ovarian cancer clinical samples. (A) Relative mRNA expression level of AQP1 in 46 cases of ovarian cancer and 10 normal ovarian tissues were determined by reverse transcription-quantitative polymerase chain reaction. GAPDH served as an internal control. (B) AQP1 protein expression was determined in ovarian cancer and normal ovarian tissue by immunohistochemical staining. ${ }^{* *} \mathrm{P}<0.01$. AQP1, aquaporin.

at $37^{\circ} \mathrm{C}$ and were then transfected with si-NC or si-AQP1 at $100 \mathrm{nM}$ for $48 \mathrm{~h}$. Following this, the cells were harvested by trypsinization, washed in ice-cold PBS, and fixed in ice-cold ethanol in PBS for 15 min. Bovine pancreatic RNase (Sigma-Aldrich; Merck KGaA) was added to each well at a final concentration of $2 \mathrm{mg} / \mathrm{ml}$, and cells were cultured at $37^{\circ} \mathrm{C}$ for $4 \mathrm{~h}$ in a $5 \% \mathrm{CO}_{2}$ incubator for $30 \mathrm{~min}$. Cell apoptosis was determined using an Annexin V Apoptosis Detection kit (Invitrogen; Thermo Fisher Scientific, Inc.) according to the manufacturer's protocol, using a FACS Calibur flow cytometer (BD Biosciences, Franklin Lakes, NJ, USA). The apoptosis ratio was calculated using CellQuest software version 2.8 (BD Biosciences).

Wound healing. Cell migration was determined using a wound healing assay. Briefly, the transfected cells $\left(5 \times 10^{4}\right)$ were seeded on a 24 -well plate and allowed to reach confluence. Following this, an artificial homogenous wound was scratched into the monolayer using a sterile plastic micropipette tip. The monolayer of cells was washed three times with PBS $(\mathrm{pH}=7.2)$ to remove the detached cells. The remaining adherent cells were cultured in RPMI-1640 medium supplemented with 10\% FBS at $37^{\circ} \mathrm{C}$ for $24 \mathrm{~h}$. Cells were imaged at 0 and $24 \mathrm{~h}$ following the wounding under an inverted phase-contrast microscope (Leica Microsystems GmbH, Wetzlar, Germany). The migration index was calculated at 5 randomly selected fields using the following formula: Migration index = number of migrated cells in experimental group/Number of migrated cells in control group (si-NC group).

Invasion assay. The invasive properties of cells were determined using Transwell chamber inserts (EMD Millipore) coated with Matrigel according to the manufacturer's protocol. Briefly, $5 \times 10^{4}$ transfected cells were added to the top chamber of inserts with Matrigel (BD Biosciences) and cultured in serum-free RPMI-1640 medium. The lower chamber was filled with RPMI-1640 medium supplemented with 10\% FBS as a chemoattractant. Following $48 \mathrm{~h}$ incubation, cells on the surface of the upper chamber were removed by scraping with a cotton swab, and the cells that had invaded the lower chamber of the filter were fixed with $70 \%$ ethanol for $30 \mathrm{~min}$ at room temperature and stained with $0.2 \%$ crystal violet for $10 \mathrm{~min}$ at room temperature. Photomicrographs of 5 randomly selected fields of the fixed cells were captured and viable cells were counted under a phase-contrast microscope (Olympus Corp.).

Statistical analysis. All data are presented as the mean \pm standard deviation of at least 3 independent experiments. Statistical analysis was performed using SPSS software version 16.0 (SPSS Inc., Chicago, IL, USA). The statistical significance of the differences between groups was assessed using a Student's t-test for pair-wise comparisons or a one-way analysis of variance followed by a post hoc Tukey's test for multiple comparisons. $\mathrm{P}<0.05$ was considered to indicate a statistically significant difference.

\section{Results}

AQP1 is upregulated in ovarian cancer and correlates with clinical features of patients with ovarian cancer. To identify the potential roles of AQP1 in the development and progression of ovarian cancer, the present study determined its mRNA and protein expression level in 46 ovarian cancer tissues and 10 ovarian tissues using RT-qPCR and immunohistochemistry, respectively. The results of qPCR demonstrated that mRNA expression levels of AQP1 were significantly higher in ovarian tumors compared with their normal tissue counterparts $(\mathrm{P}<0.01$; Fig. 1A). Elevated protein levels of AQP1 were observed in ovarian tumors compared with the normal ovarian tissues as demonstrated by immunochemical staining (Fig. 1B).

AQP1 expression in ovarian cancer cells is inhibited by $s i-A Q P 1$. To study the biological role of AQP1 in ovarian cancer progression, the present study transfected AQP1 siRNA (si-AQP1) or scramble siRNA (si-NC) into human ovarian cancer SKOV3 cells, which were then cultured for $48 \mathrm{~h}$. Following this, the mRNA and protein levels of AQP1 were analyzed with RT-qPCR and western blotting, respectively. As presented in Fig. 2A and B, AQP1 expression at the mRNA and protein level was significantly inhibited in SKOV3 cells 
A

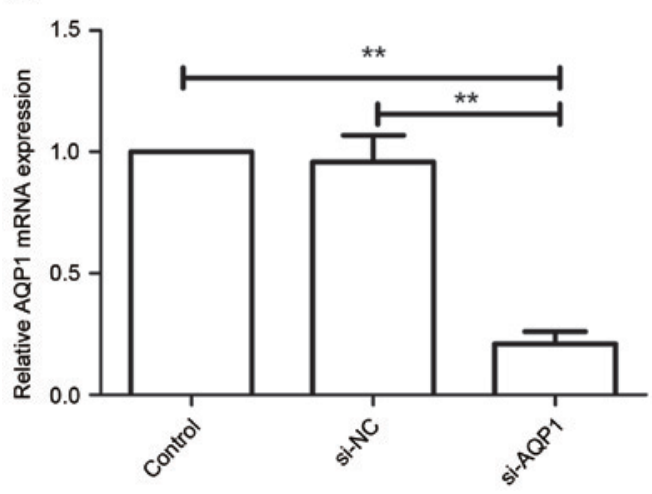

B

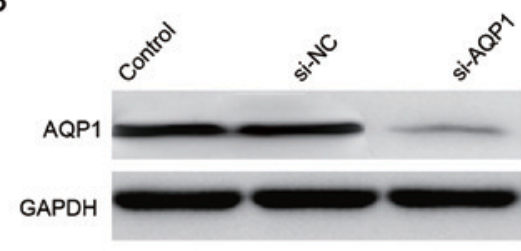

Figure 2. AQP1 expression in ovarian cancer cells is inhibited by si-AQP1. (A) Relative mRNA expression level of AQP1 was determined in SKOV3 cells transfected with si-AQP1 or si-NC by reverse transcription-quantitative polymerase chain reaction. GAPDH served as an internal control. (B) AQP1 protein expression was determined in SKOV3 cells transfected with si-AQP1 or si-NC by western blotting. GAPDH served as an internal control. ${ }^{* *} \mathrm{P}<0.01$. AQP1, aquaporin; si, small interfering; $\mathrm{NC}$, negative control.

A

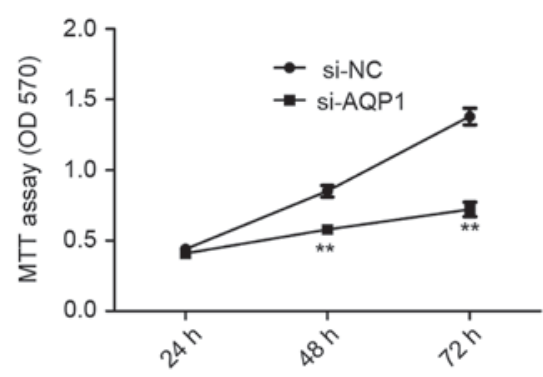

B

$\bar{\alpha}$

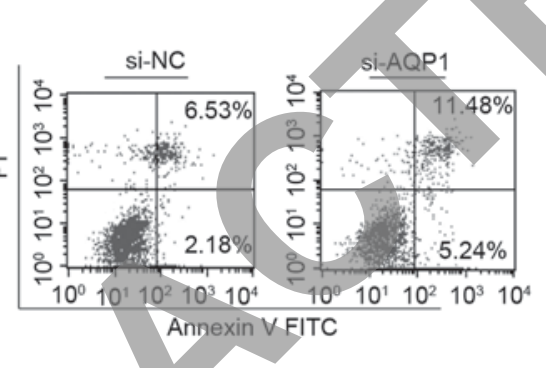

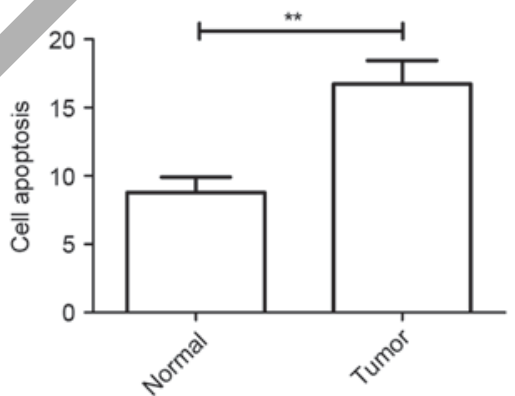

Figure 3. Downregulation of AQP1 decreases cell viability and induces cell apoptosis in ovarian cancer cells. (A) Cell viability was determined in SKOV3 cells transfected with si-AQP1 or si-NC by an MTT assay. (B) Cell apoptosis was determined in SKOV3 cells transfected with si-AQP1 or si-NC by flow cytometry. ${ }^{* *} \mathrm{P}<0.01$ vs. si-NC. AQP1, aquaporin; si, small interfering; NC, negative control; OD, optical density; FITC, fluorescein isothiocyanate; PI, propidium iodide.
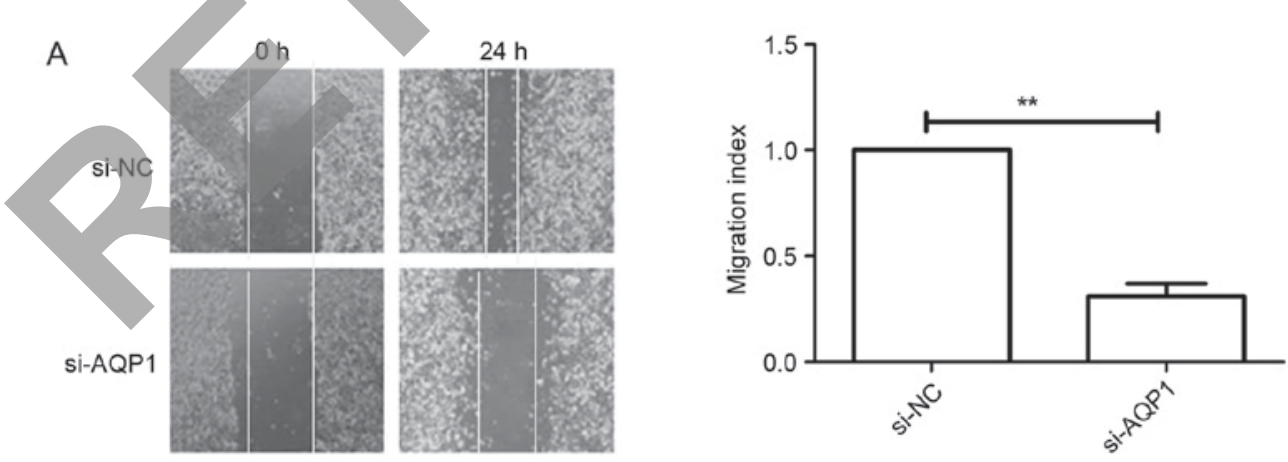

B

si-NC

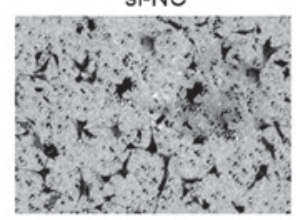

si-AQP1

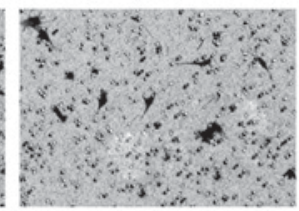

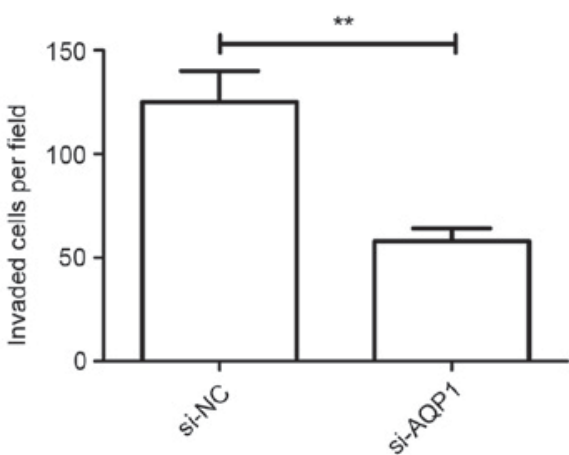

Figure 4. Downregulation of AQP1 inhibits cell migration and invasion in ovarian cancer cells. (A) Cell migration was determined in SKOV3 cells transfected with si-AQP1 or si-NC by a wound healing assay. (B) Cell invasion was determined in SKOV3 cells transfected with si-AQP1 or si-NC by a Transwell invasion chamber assay. ${ }^{* *} \mathrm{P}<0.01$. AQP1, aquaporin; si, small interfering; $\mathrm{NC}$, negative control. 
transfected with si-AQP1 compared with cells transfected with si-NC $(\mathrm{P}<0.01)$.

Downregulation of AQP1 decreases cell viability and induces cell apoptosis in ovarian cancer cells. To investigate the effect of AQP1 on cell viability, an MTT assay was performed. It was demonstrated that downregulation of AQP1 in SKOV3 cells resulted in a notable decrease in cell viability $(\mathrm{P}<0.01$; Fig. 3A). In addition, the effects of AQP1 on apoptosis in ovarian cancer cells were assessed. As presented in Fig. 3B, downregulation of AQP1 in SKOV3 cells significantly induced cell apoptosis compared with si-NC group $(\mathrm{P}<0.01)$.

Downregulation of AQP1 inhibits cell migration and invasion in ovarian cancer cells. To investigate if AQP1 effected migration and invasion in SKOV3 cells, a wound healing and transwell assay were performed. It was demonstrated that downregulation of AQP1 significantly suppressed migration (Fig. 4A) and invasion (Fig. 4B) in ovarian cancer cells compared with si-NC group.

\section{Discussion}

The clinical data of the present study demonstrated that AQP1 expression was increased at the mRNA and protein level in ovarian cancer tissues, which was in accordance with the results of a previous study (21). The in vitro experiments demonstrated that downregulation of AQP1 by siRNA in ovarian cancer cells significantly inhibited cell proliferation, migration and invasion, and induced cell apoptosis. These results suggested that AQP1 may serve as a useful diagnostic marker and potential target for the treatment of ovarian cancer.

AQP1 has previously been demonstrated to be overexpressed in various tumors, and is important in tumor progression in numerous types of cancers (16-22). AQP1 has been reported to be overexpressed in malignant tumors of the lung, however is not present in normal lung tissue $(24,25)$. Notably, the upregulation of AQP1 in micropalliary adenocarcinoma patients is associated with a poor survival rate, indicating the participation of AQP1 in the spread of the micropalliary component (25). In AQP1-null and wide type mice, implantation of B16F10 melanoma cells demonstrates impaired tumor growth in AQP1-null mice due to failure in angiogenesis (12). Another investigation suggested that AQP1 induces tumor angiogenesis via activating the $\operatorname{Lin} 7 / \beta$-catenin signaling pathways (26). Using in situ hybridization, Moon et al (27) demonstrated that during tumorigenesis of colorectal cancer, the expression levels of AQP1 and AQP5 are upregulated from an early stage, and maintained until the late stage of cancer development. Significantly elevated expression levels of AQP1 are additionally present in proliferating cancerous microvessels in rats (28) and humans (29). Further data from AQP1 knock-out mice and forced-expression cancer cell lines suggest that tumor cell migration is partly due to AQP1-dependent water permeability into cellular protrusions, which help to create more space for actin polymerization at the leading margin of migrating tumor cells $(10,29,30)$. Furthermore, patients with ascites $>1,000 \mathrm{ml}$ demonstrate upregulation of AQP1 (31). Wu et al (22) demonstrated that AQP1 knockdown may effectively inhibit cell proliferation, adhesion, invasion and tumorigenesis by targeting the tumor growth factor- $\beta$ signaling pathway and focal adhesion genes. A previous study demonstrated that AQP1 is upregulated in ovarian cancer tissues compared with normal ovarian tissues (21), however the specific functional role of AQP1 in ovarian cancer remains to be fully elucidated. The present study demonstrated that downregulation of AQP1 in ovarian cancer cells reduced cell viability, migration and invasion, and induced cell apoptosis in vitro, suggesting that AQP1 is involved in the progression of ovarian cancer.

In conclusion, the present study demonstrated that AQP1 was elevated in the majority of ovarian cancer tissues. Notably, the present study, to the best of our knowledge, suggests for the first time that AQP1 exhibits a key role in the viability, apoptosis, migration and inyasion of ovarian cancer cells. These findings indicate that AQP1 may serve as a useful diagnostic marker and potential target for the treatment of ovarian cancer in the future.

\section{References}

1. Siegel R, Naishadham D and Jemal A: Cancer statistics, 2013. CA Cancer J Clin 63: 11-30, 2013.

Permuth-Wey J and Sellers TA: Epidemiology of ovarian cancer. Methods Mol Biol 472: 413-437, 2009.

3. Dembo AJ, Davy M, Stenwig AE, Berle EJ, Bush RS and Kjorstad K: Prognostic factors in patients with stage I epithelial ovarian cancer. Obstet Gynecol 75: 263-273, 1990.

4. Fastaia $\mathbf{J}$ and Dumont AE: Pathogenesis of ascites in mice with peritoneal carcinomatosis. J Natl Cancer Inst 56: 547-550, 1976.

Tamsma J: The pathogenesis of malignant ascites. Cancer Treat Res 134: 109-118, 2007.

6. Saada E, Follana P, Peyrade F, Mari V and Francois E: Pathogenesis and management of refractory malignant ascites. Bull Cancer 98: 679-687, 2011 (In French).

7. Nico B and Ribatti D: Aquaporins in tumor growth and angiogenesis. Cancer Lett 294: 135-138, 2010.

8. Mobasheri A and Barrett-Jolley R: Aquaporin water channels in the mammary gland: From physiology to pathophysiology and neoplasia. J Mammary Gland Biol Neoplasia 19: 91-102, 2014.

9. Papadopoulos MC, Saadoun S and Verkman AS: Aquaporins and cell migration. Pflugers Arch 456: 693-700, 2008.

10. Verkman AS: Aquaporin water channels and endothelial cell function. J Anat 200: 617-627, 2002.

11. Connolly DL, Shanahan CM and Weissberg PL: The aquaporins. A family of water channel proteins. Int J Biochem Cell Biol 30: 169-172, 1998.

12. Saadoun S, Papadopoulos MC, Hara-Chikuma M and Verkman AS: Impairment of angiogenesis and cell migration by targeted aquaporin-1 gene disruption. Nature 434: 786-792, 2005.

13. Papadopoulos MC, Saadoun S, Davies DC and Bell BA: Emerging molecular mechanisms of brain tumouroedema. Br J Neurosurg 15: 101-108, 2001.

14. Bai C, Fukuda N, Song Y, Ma T, Matthay MA and Verkman AS: Lung fluid transport in aquaporin-1 and aquaporin-4 knockout mice. J Clin Invest 103: 555-561, 1999.

15. King LS, Nielsen S and Agre P: Aquaporin-1 water channel protein in lung: Ontogeny, steroid-induced expression, and distribution in rat. J Clin Invest 97: 2183-2191, 1996.

16. Chen Y, Tachibana O, Oda M, Xu R, Hamada J, Yamashita J, Hashimoto $\mathrm{N}$ and Takahashi JA: Increased expression of aquaporin 1 in human hemangioblastomas and its correlation with cyst formation. J Neurooncol 80: 219-225, 2006.

17. El Hindy N, Bankfalvi A, Herring A, Adamzik M, Lambertz N, Zhu Y, Siffert W, Sure U and Sandalcioglu IE: Correlation of aquaporin-1 water channel protein expression with tumor angiogenesis in human astrocytoma. Anticancer Res 33: 609-613, 2013.

18. Mobasheri A, Airley R,Hewitt SM and Marples D: Heterogeneous expression of the aquaporin 1 (AQP1) water channel in tumors of the prostate, breast, ovary, colon and lung: A study using high density multiple human tumor tissue microarrays. Int J Oncol 26: $1149-1158,2005$ 
19. Oshio K, Binder DK, Liang Y, Bollen A, Feuerstein B, Berger MS and Manley GT: Expression of the aquaporin-1 water channel in human glial tumors. Neurosurgery 56: 375-381, 2005.

20. Saadoun S, Papadopoulos MC, Davies DC, Bell BA and Krishna S: Increased aquaporin 1 water channel expression in human brain tumours. Br J Cancer 87: 621-623, 2002.

21. Takal MK, Baykal C, Baser E, Kaya MD, Dursun P, Ozen O, Haberal AN and Ayhan A: Does Aquaporin-1 expression have clinical significance in serous epithelial ovarian cancer? J Turk Ger Gynecol Assoc 14: 130-135, 2013.

22. Wu Z, Li S, Liu J, Shi Y, Wang J, Chen D, Luo L, Qian Y, Huang X and Wang H: RNAi-mediated silencing of AQP1 expression inhibited the proliferation, invasion and tumorigenesis of osteosarcoma cells. Cancer Biol Ther 16: 1332-1340, 2015.

23. Livak KJ and Schmittgen TD: Analysis of relative gene expression data using real-time quantitative PCR and the 2(-Delta Delta C(T)) method. Methods 25: 402-408, 2001.

24. Hoque MO, Soria JC, Woo J, Lee T, Lee J, Jang SJ, Upadhyay S, Trink B, Monitto C, Desmaze C, et al: Aquaporin 1 is overexpressed in lung cancer and stimulates NIH-3T3 cell proliferation and anchorage-independent growth. Am J Pathol 168: 1345-1353, 2006.

25. Machida Y, Ueda Y, Shimasaki M, Sato K, Sagawa M, Katsuda S and Sakuma T: Relationship of aquaporin 1,3 and 5 expression in lung cancer cells to cellular differentiation, invasive growth, and metastasis potential. Hum Pathol 42: 669-678, 2011
26. Monzani E, Bazzotti R, Perego $\mathrm{C}$ and La Porta CA: AQP1 is not only a water channel: It contributes to cell migration through Lin7/beta-catenin. PLoS One 4: e6167, 2009.

27. Moon C, Soria JC, Jang SJ, Lee J, Obaidul Hoque M, Sibony M, Trink B, Chang YS, Sidransky D and Mao L: Involvement of aquaporins in colorectal carcinogenesis. Oncogene 22: 6699-6703, 2003.

28. Endo M, Jain RK, Witwer B and Brown D: Water channel (aquaporin 1) expression and distribution in mammary carcinomas and glioblastomas. Microvasc Res 58: 89-98, 1999.

29. Verkman AS: Knock-out models reveal new aquaporin functions. Handb Exp Pharmacol: 359-381, 2009.

30. Verkman AS, Hara-Chikuma $\mathrm{M}$ and Papadopoulos MC: Aquaporins-new players in cancer biology. J Mol Med (Berl) 86 523-529, 2008

31. Yang JH, Yu YQ and Yan CX: Localisation and expression of aquaporin subtypes in epithelial ovarian tumours. Histol Histopathol 26: 1197-1205, 2011. 